\title{
Modulation of Prepulse Inhibition and Startle Reflex by Emotions: A Comparison between Young and Older Adults
}

\author{
Jolyanne Le Duc ${ }^{1,2,3 \dagger}$, Philippe Fournier ${ }^{1,2,3 \dagger}$ and Sylvie Hébert ${ }^{1,2,3 *}$ \\ ${ }^{1}$ School of Speech Pathology and Audiology, Faculty of Medicine, Université de Montréal, Montréal, QC, Canada, \\ ${ }^{2}$ BRAMS, International Laboratory for Research on Brain, Music, and Sound, Université de Montréal, Montréal, QC, \\ Canada, ${ }^{3}$ CRIUGM, Centre de Recherche de I'Institut Universitaire de Gériatrie de Montréal, Montréal, QC, Canada
}

This study examined whether or not the acoustic startle response and sensorimotor gating may be modulated by emotions differentially between young and older adults. Two groups of participants (mean age Young: 24 years old; Elderly: 63.6 years old) were presented with three types of auditory stimuli (Startle alone, High or Low frequency Prepulse) while viewing pleasant, neutral, or unpleasant images. Electromyographic activity of the eyeblink response was measured. Results show that older adults displayed diminished eyeblink responses whereas younger adults displayed enhanced eyeblink responses when viewing negative images. Sensorimotor gating also differed between young and older adults, with enhanced sensorimotor gating abilities while viewing

OPEN ACCESS

Edited by:

P. Hemachandra Reddy,

Texas Tech University, USA

Reviewed by:

Erica Duncan,

Emory University, USA

Mara Mather,

University of Southern California, USA

*Correspondence:

Sylvie Hébert

sylvie.hebert@umontreal.ca

tThese authors have contributed equally to this work.

Received: 06 August 2015 Accepted: 09 February 2016 Published: 23 February 2016

Citation:

Le Duc J, Fournier $P$ and Hébert $S$ (2016) Modulation of Prepulse Inhibition and Startle Reflex by

Emotions: A Comparison between Young and Older Adults.

Front. Aging Neurosci. 8:33. doi: 10.3389/fnagi.2016.00033 positive pictures in older adults and diminished abilities while viewing negative pictures among younger adults. These results argue in favor of a differential emotional influence on the sensorimotor abilities of young and older adults, with a positivity bias among the latter.

Keywords: acoustic startle reflex, prepulse inhibition, aging, young, emotions, international affective picture system, eyeblink, positivity effect

\section{INTRODUCTION}

The acoustic startle reflex is a cross-species, whole-body reflex in response to a loud and unexpected sound. Its basic circuitry is relatively simple (Cook et al., 1991; Kofler et al., 2001) and it is easily measured in humans by the magnitude and latency of the eyeblink, one component of the startle response. The presentation of a soft, non-startling sound (a "prepulse") 30-500 ms before the startling sound reduces the startle reflex (Braff et al., 2001). Prepulse inhibition (PPI) reflects a fundamental protective function by reducing disruptive influences to the processing of prepulse signals, and is impaired in various cognitive disorders such as schizophrenia (Braff et al., 1978). It is widely acknowledged that the PPI paradigm provides an operational measure of pre-attentive sensorimotor gating. PPI itself can be modulated via descending projections from central brain structures such as auditory cortex and limbic circuitry (Li et al., 2009). This modulation determines how strongly the prepulse will inhibit the subsequent neural response from the facial motor nucleus responsible of the startle response (Swerdlow et al., 2001). The purpose of the present study was to characterize the interaction between emotion and aging on the startle reflex and its inhibition by a prepulse. 
The general consensus among the many studies investigating the influence of emotions on the startle reflex is that it is reliably modulated by the affective valence of the emotions, i.e., pleasant vs. unpleasant (Vrana et al., 1988; Bradley et al., 1990, 1996b; Lang et al., 1990; Lang, 1995; Vanman et al., 1996; Hawk and Cook, 1997, 2000; Bradley and Lang, 2000; Roy et al., 2009), including fear (for a review, see Grillon and Baas, 2003). When exposed to pleasant, neutral or unpleasant stimuli (pictures or sounds), young adults show a typical pattern consisting of a stronger startle reflex during unpleasant than under neutral emotion, and a weaker one during pleasant than under neutral condition (Vrana et al., 1988; Bradley et al., 1990, 1993, 1996a,c; Feng et al., 2011). This pattern of responses is interpreted in terms of appetitive vs. aversive states, negative affective states engaging the defensive system that entails potentiation of the startle reflex, and positive affective states engaging the appetitive system that diminishes the startle reflex (Lang et al., 1990).

When considering the influence of age and emotion on the startle reflex, the appetitive/aversive model does not apply in a straightforward fashion. Accordingly, a recent study reported opposite eyeblink patterns for young and old adults: young adults displayed the typical pattern, that is, potentiated eyeblink when viewing unpleasant pictures whereas older adults displayed a reduced eyeblink when viewing the same pictures (Feng et al., 2011).

The possibility of a joint influence of emotions and age on PPI is important to consider since PPI is widely used to investigate pathological conditions such as schizophrenia-usually encountered in young people-and Alzheimer's disease-found in the elderly (Braff and Geyer, 1990; Kumari et al., 2007; Wang et al., 2012). If sensorimotor gating were influenced by affective states or aging, these might become confounding variables in the analysis of PPI deficits. In the present study, young and older adults were asked to view pleasant, neutral or unpleasant pictures retrieved from the International Affective Picture System (IAPS; Bradley and Lang, 2007) paired with acoustic startle and prepulse sounds. Based on previous work (Feng et al., 2011), our expectation was that negative pictures would potentiate the startle reflex response in the young but not in the elderly. Given the paucity of available information regarding the influence of age and emotion on PPI (e.g., Hawk and Cook, 2000, who found no effect of emotions on PPI in young adults), our only expectation was that-if positive and negative emotions are processed differently in young and older adults-, PPI in older adults should be different for positive compared to neutral and negative images, and either the opposite pattern or no effect of emotions in young adults.

\section{MATERIALS AND METHODS}

\section{Participants}

Two groups participated in the study: 26 Young (mean age of 24 years; range $=20-29, \mathrm{SD}=2.6$ ) and 29 Elderly adults (mean age of 63.6 years; range $=56-69, \mathrm{SD}=3.6$ ).
An additional 7 young and 17 elderly adults were tested but were eliminated because they were considered non-responsive to the startle paradigm (see "Data Processing" Section below for details). Four young and one elderly participants were excluded on the basis of noisy EMGs or too many spontaneous blinks just before the startling stimuli. Participants were recruited through posted ads, word of mouth, as well as BRAMS' and CRIUGM's participant pools. The two groups were similar in gender $(9$ men in the Young and 10 in the Elderly group) and depressive symptomatology, but the younger participants were slightly more educated than the elderly (Table 1). Preliminary analyses with years of education as a covariate did not yield any significant effect of this factor or interaction with any other factors. They all selfreported good psychological and physical health and had normal hearing or only mild hearing loss as confirmed by audiometry performed in the laboratory with clinical equipment and procedure (hearing thresholds $<45 \mathrm{~dB} \mathrm{HL}$ at any frequency between $250 \mathrm{~Hz}$ and $4 \mathrm{kHz}$ in at least one ear) and normal or corrected-to-normal vision. Participants with uncontrolled medical conditions (e.g., hypertension, diabetes), psychological, psychiatric or neurological conditions (depression, mood disorders, anxiety, bipolarity, etc.) and medication intake affecting nervous system were excluded. None of the participants was a smoker. The study was approved by the local ethics committees of the Université de Montréal and Institut Universitaire de Gériatrie de Montréal and was conducted with informed and written consent from all participants.

\section{Stimuli and Apparatus}

\section{Questionnaire}

The Beck Depression Inventory II (BDI-II; Beck et al., 1996) was used to rule out participants suffering from severe depression (scores $\geq 30$; Cook et al., 1991).

\section{Startle and PPI Stimuli}

As previously reported (Fournier and Hébert, 2013), the auditory task consisted of three different stimuli (Figure 1): (i) Startle, a $50 \mathrm{~ms}$ broadband noise burst $(20 \mathrm{~Hz}-20 \mathrm{kHz})$ set at $105 \mathrm{~dB}(\mathrm{~A})$ SPL with near instantaneous rise-fall time $(<1 \mathrm{~ms})$; (ii) low frequency prepulses centered at $500 \mathrm{~Hz}(200-1200 \mathrm{~Hz})$; and (iii) high-frequency prepulses centered at $4000 \mathrm{~Hz}(3.5-4.5 \mathrm{kHz})$. Two different prepulse sounds were used in order to maximize the probability of producing inhibition. The prepulse trials were $50 \mathrm{~ms}$ noise bursts presented at $65 \mathrm{~dB}(\mathrm{~A}) \mathrm{SPL}$ with near instantaneous rise-fall time $(<1 \mathrm{~ms})$ that preceded a startle noise by $120 \mathrm{~ms}$. The inter-stimulus interval (ISI) of $120 \mathrm{~ms}$ (SOA = $170 \mathrm{~ms}$ ) was selected to maximize magnitude inhibition (Braff et al., 1978). The Intertrial-interval (ITI) time was randomly set at a value between 15 and $23 \mathrm{~s}$ in each block to decrease anticipation of the auditory stimulus (Patrick et al., 1993). All stimuli were presented in a silent background and created using Max/MSP Software program (Cycling 74, San Francisco, CA, USA). 
TABLE 1 | Socio-demographic characteristics (standard deviation, SD) of Young adults and Elderly.

\begin{tabular}{|c|c|c|c|}
\hline & Young $(N=26)$ & Elderly $(N=29)$ & $p$-value \\
\hline Age in years (SD) & $24.0(2.6)$ & $63.6(3.6)$ & $<0.001$ \\
\hline Male/Female & $9 / 17$ & $10 / 19$ & n.s. \\
\hline Education in years (SD) & $16.5(1.8)$ & $14.8(3.1)$ & 0.02 \\
\hline BDI-II (SD) & $5.3(4.0)$ & $4.6(5.3)$ & n.s. \\
\hline \multicolumn{4}{|l|}{ Right ear } \\
\hline PTA low frequencies & $6.3(4.7)$ & $9.4(7.2)$ & n.s. \\
\hline PTA mid frequencies & $5.7(4.5)$ & $13.9(10.7)$ & 0.001 \\
\hline PTA high frequencies & $4.8(5.0)$ & $28.4(13.5)$ & $<0.001$ \\
\hline \multicolumn{4}{|l|}{ Left ear } \\
\hline PTA low frequencies & $3.9(4.7)$ & $9.1(7.1)$ & 0.002 \\
\hline PTA mid frequencies & $4.1(4.9)$ & $11.6(7.0)$ & $<0.001$ \\
\hline PTA high frequencies & $5.0(4.9)$ & $28.1(13.4)$ & $<0.001$ \\
\hline
\end{tabular}

Pure tone threshold average (PTA) in dB HL (SD) for low frequencies (250, 500, $750 \mathrm{~Hz})$, mid frequencies (1000, 1500, 2000 Hz) and high frequencies (3000, 4000, 6000, $8000 \mathrm{~Hz}$ )

\section{Visual Stimuli}

Originally 75 color photographs representing daily objects or situations that elicit emotions normally occurring in life were selected from the International Affective Picture System (Bradley and Lang, 2007). A pilot study was conducted involving 30 young (19-27 years old) and 27 older participants (55-80 years old) not included in this study but recruited using the same inclusion and exclusion criteria, in order to create two sets of stimuli comparable for the Young and the Elderly groups, as assessed with the Self-Assessment Manikin (SAM; Bradley et al., 1993; Bradley and Lang, 1994). Pictures were pleasant, neutral or unpleasant. To minimize the confounding effect of arousal on physiological responses, a subset of stimuli were chosen such that pictures from each emotional category did not differ in level of arousal between age groups. The final set thus included 66 pictures (22 pictures for each emotional category), with 59 pictures common to both groups and seven that differed across groups. While arousal ratings did not differ between age groups for any emotional category $(F s<1$ for both Group and Group $\times$ Emotion), the corresponding

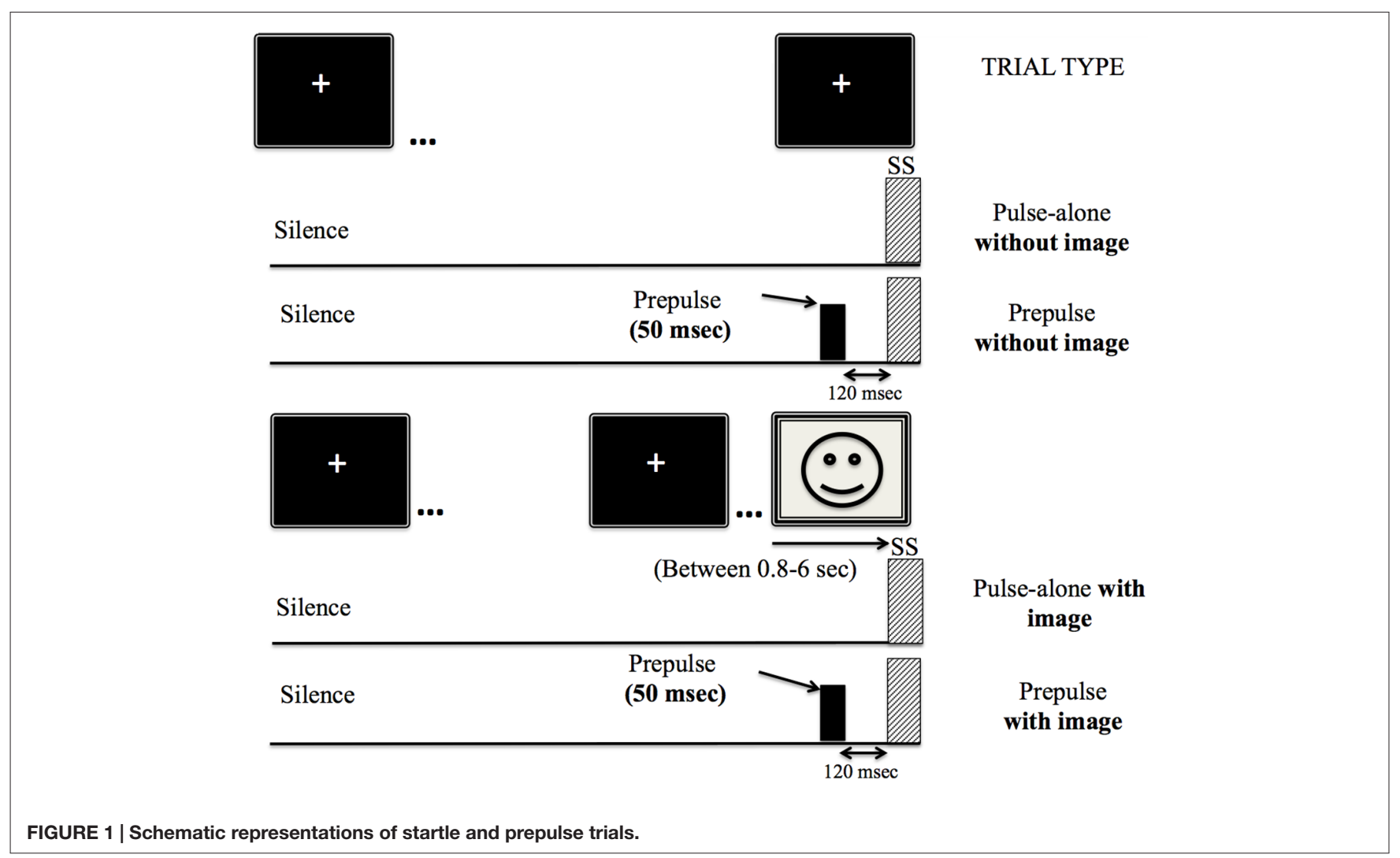


valence, however, differed. Like several previous studies have reported (Grühn and Scheibe, 2008), older adults rated positive pictures as more positive than younger adults (with means of 7.93 and 7.13, respectively, $p<0.001$ ), and negative pictures as more negative (with means of 1.79 and 2.19, respectively, $p<0.03$ ). All pictures numbers are available upon request.

\section{EMG Measurement}

Eyeblink activity was measured using two $4 \mathrm{~mm} \mathrm{Ag} / \mathrm{AgCl}$ shielded recording electrodes positioned $1.5 \mathrm{~cm}$ apart on the orbicularis oculi muscle under the left eye and a ground electrode on the forehead, according to guidelines (Blumenthal et al., 2005). Signal acquisition was made using a IMac running the Acqknowledge 4.1 Software connected to a Biopac MP150 System (Biopac Systems, Inc., Santa Barbara, CA, USA) using the following settings: $1000 \times$ amplification, $90-500 \mathrm{~Hz}$ bandpass filter, RMS transformation, A/D conversion at $1 \mathrm{kHz}$. The stimulus presentation system was coupled to a Fireface sound card (RME, Haimhausen, Germany) hosted by a PC computer. Startle noise presentation was synchronized with eyeblink activity recording via a square-wave trigger signal to precisely determine the window of responses for magnitudes and latencies of the eyeblink (see "Data Processing" Section below).

\section{Procedure}

Participants were instructed to sit quietly in a soundproof booth, refrain from moving and listen to the sounds presented binaurally via closed dynamic headphones DT 770 Pro/250 while watching a series of pictures projected on a dark screen. The test session began with a 2 min silent acclimatizing period followed by practice trials (four pulse-alone stimuli and one picture of each type). The task itself consisted of three equivalent blocks of trials of an approximately 8-min duration counterbalanced for pleasantness and arousal. Each block consisted of 21 auditory stimuli (seven startles, seven low-frequency and seven high frequency prepulses) and 21 pictures (seven pleasant, seven neutral and seven unpleasant). Six pictures of each type were pseudo-randomly combined with an auditory stimulus (e.g., two pleasant pictures with startle, two pleasant pictures with low-frequency prepulse, two pleasant pictures with high frequency prepulse per block). One auditory stimulus of each type was presented without any image for control purposes and one image of each type was presented without auditory stimulus to reduce predictability. Within each block, images were pseudo-randomly presented such that no more than two images with the same picture type (pleasant, neutral and unpleasant) were presented in succession. Participants viewed each image for $6 \mathrm{~s}$, and the auditory stimulus arose randomly between $800 \mathrm{~ms}$ and $6 \mathrm{~s}$ after image onset. The first $800 \mathrm{~ms}$ were excluded since picture onset is known to cause PPI and reflect attention processes (Bradley et al., 1993). All stimulus types were calibrated before each testing session with an SE SoundPro DL 1/3 octave level meter (Quest Technologies, WI, USA) using an EC-9A artificial ear coupler (Quest Electronics, Oconomowoc, WI, USA) with appropriate rates, that is, impulse for startle noises/prepulse, using the A-weighting frequency curve. The total duration of the task was about $30 \mathrm{~min}$.

\section{Data Processing and Statistical Analyses}

For valence and arousal ratings in the pilot study, data above two $\mathrm{SD}$ from the group mean were replaced by the average value of the appropriate group (Young or Elderly) for each picture type $(6.6 \%)$. Statistical analyses were run separately on valence and arousal. Data were entered into analysis of variances (ANOVAs) with Emotional condition (Unpleasant, Neutral, Pleasant) as within-subjects factors and Group (Young vs. Elderly) as the between-subject factor.

\section{Startle and PPI Stimuli}

All trials were visually inspected for excessive noise in the EMG signal and for any spontaneous blink occurring immediately before the startle stimuli. These trials were few $(8.1 \%$ for Young adults and $6.8 \%$ for Elderly) and rejected from further analysis. For each participant, a baseline was defined from startle-alone trials only, by averaging the highest RMS amplitude value within a $200 \mathrm{~ms}$ window occurring 9-17 s before the startle noise onset, i.e., while no picture viewing. The peak-topeak amplitude of each startle response 20-120 ms from pulse onset was extracted from the RMS data. Data for each trial type were averaged for each picture type for each participant. Any peak-to-peak amplitude value of any trial (i.e., prepulse or startle) $<2$ SD above the average baseline was considered a non-response and was assigned zero magnitude. In addition, only participants having a $100 \%$ response probability for startle trials were included in the study (final sample $=26$ young and 29 elderly).

For raw EMG startle, data were entered in a mixed ANOVA with Emotional condition (Unpleasant, Neutral, Pleasant) and Group (Young vs. Elderly) as the between-subject factor. For Startle and PPI, $Z$-scores were also calculated to account for high levels of variability between groups in EMG responses (Patrick et al., 1993; Cuthbert et al., 1996; Feng et al., 2011). Startle and PPI $Z$-scores data $>2 \mathrm{SD}$ from the group mean were replaced by the average value of the appropriate group (Young, Elderly) for each picture type (representing only $4.8 \%$ of all data). $Z$-scores were calculated for each participant using the formula $z=(x-\bar{x}) / s$, where $x$ is the magnitude value of the trial, $\bar{x}$ is the mean response magnitude for all trials and $s$ is the standard deviation of all trials.

Peak latency was obtained from the same time window but calculated from the raw EMG waveform following guidelines (Blumenthal et al., 2005). Raw latency data for startle and for PPI trials were entered in two separate mixed ANOVAs with Emotional Condition (Unpleasant, Neutral, Pleasant) as the within-subject factor and Group (Young vs. Elderly) as the between-subject factor. Latency facilitation was also calculated for each condition (Low- or High-Frequency prepulse) using the following formula: Latency facilitation = (pulsealone latency) - (Low- or High-Frequency prepulse) latency. Latency facilitation data were entered in a mixed ANOVA with Emotional Condition (Unpleasant, Neutral, Pleasant) and 
Stimuli type (PPI High frequency, PPI Low frequency) as the within-subject factors and Group (Young vs. Elderly) as the between-subject factor. Peak rather than onset latencies were calculated because unlike onset latencies, peak latencies are not confounded by changes in reflex magnitude (Cadenhead et al., 1999).

All significant interactions and main effects were followed by appropriate ANOVA or $t$-tests. Bonferroni's correction for multiple comparisons was used for $t$-tests when appropriate in order to keep the alpha level to 0.05 throughout all analyses, although original degrees of freedom are reported. Therefore, the $p$ values reported in this article are corrected values. Effect sizes are reported for main effects with $\eta_{p}^{2}$ (Partial Eta squared). The no-image condition was compared between groups with independent sample $t$-tests.

\section{RESULTS}

\section{Startle}

\section{Raw EMG Data}

Figure 2A displays startle raw magnitude for the two groups and the three conditions. It is easily observable that Elderly had overall much smaller blink responses $(M=61 \mu \mathrm{V}, \mathrm{SD}=$ $81 \mu \mathrm{V})$ than Young adults $(M=157 \mu \mathrm{V}, \mathrm{SD}=76 \mu \mathrm{V})$. This was supported by a main Group effect, $F_{(1,53)}=20.29, p<0.001$, $\eta_{p}^{2}=0.277$, and extends to startle trials not associated with pictures $t_{(30.4)}=3.51, p=0.001$, with means of $56 \mu \mathrm{V}(\mathrm{SD}=37)$ and $133 \mu \mathrm{V}(\mathrm{SD}=107)$, respectively.

\section{Z-Scores}

Figure 2B displays startle $Z$-scores for the two groups and the three emotional conditions. The main group effect was no longer significant, $F_{(1,58)}=2.21, p=0.09$, but the two-way interaction Emotion $\times$ Group was, $F_{(2,106)}=8.44, p<0.001, \eta_{p}^{2}=0.137$ : as expected, Young adults displayed significantly greater startle reflex reactivity for unpleasant compared to neutral pictures, $t_{(25)}=-2.98, p=0.006$, with means of $0.78(\mathrm{SD}=0.5)$ and
$0.41(\mathrm{SD}=0.7)$, respectively, and to pleasant pictures, $t_{(25)}=3.4$, $p=0.002$, with a mean of $0.35(\mathrm{SD}=0.6)$. Neutral and pleasant pictures did not differ $(t<1)$. For the Elderly, the only significant difference was between the neutral and unpleasant pictures, with lower startle reflex reactivity for unpleasant compared to neutral $t_{(29)}=2.32, p=0.034$ with means of $0.6(\mathrm{SD}=0.4)$ and 0.78 $(\mathrm{SD}=0.4)$, respectively. The difference between pleasant and unpleasant pictures failed to reach significance $t_{(29)}=-1.4$, $p=0.22$, even though the startle reactivity to pleasant pictures was higher with a mean of $0.74(\mathrm{SD}=0.5)$. Neutral and pleasant pictures did not differ $(t<1)$.

\section{Prepulse Inhibition}

\section{Z-Scores}

Figure 3 displays PPI $Z$-scores for the two groups and the three emotional conditions. The two-way interaction was significant, $F_{(2,106)}=7.23, p=0.001, \eta_{p}^{2}=0.120$. Interestingly, a significant main effect of Group was observed, $F_{(1,53)}=16.33, p<0.001$, $\eta_{p}^{2}=0.235$, with overall more inhibition in the Elderly compared to the Young group. Following up on the two-way interaction, groups were analyzed separately and frequencies were merged. For the Young adults there was a significant main effect of Emotion, $F_{(2,50)}=14.68, p<0.001, \eta_{p}^{2}=0.370$, with negative images decreasing the inhibition significantly $(M=-0.14$, $\mathrm{SD}=0.4)$ compared to positive images $t_{(25)}=-4.58, p<0.001$ $(M=-0.47, \mathrm{SD}=0.2)$ and neutral images $t_{(25)}=4.04, p<0.001$ $(M=-0.42, \mathrm{SD}=0.3)$. For the Elderly group, there was a significant main effect of Emotion as well, $F_{(2,56)}=6.55$, $p=0.003, \eta_{p}^{2}=0.190$, with more inhibition while viewing pleasant $(M=-0.6, \mathrm{SD}=0.1)$ than unpleasant $t_{(28)}=-3.76, p=0.001$ $(M=-0.5, \mathrm{SD}=0.2)$ and than neutral pictures $t_{(28)}=-2.64$, $p=0.013(M=-0.5, \mathrm{SD}=0.2)$.

\section{Latency Data}

Table 2 displays startle raw latency and latency facilitation data for the two groups in the three conditions and the without-image condition.
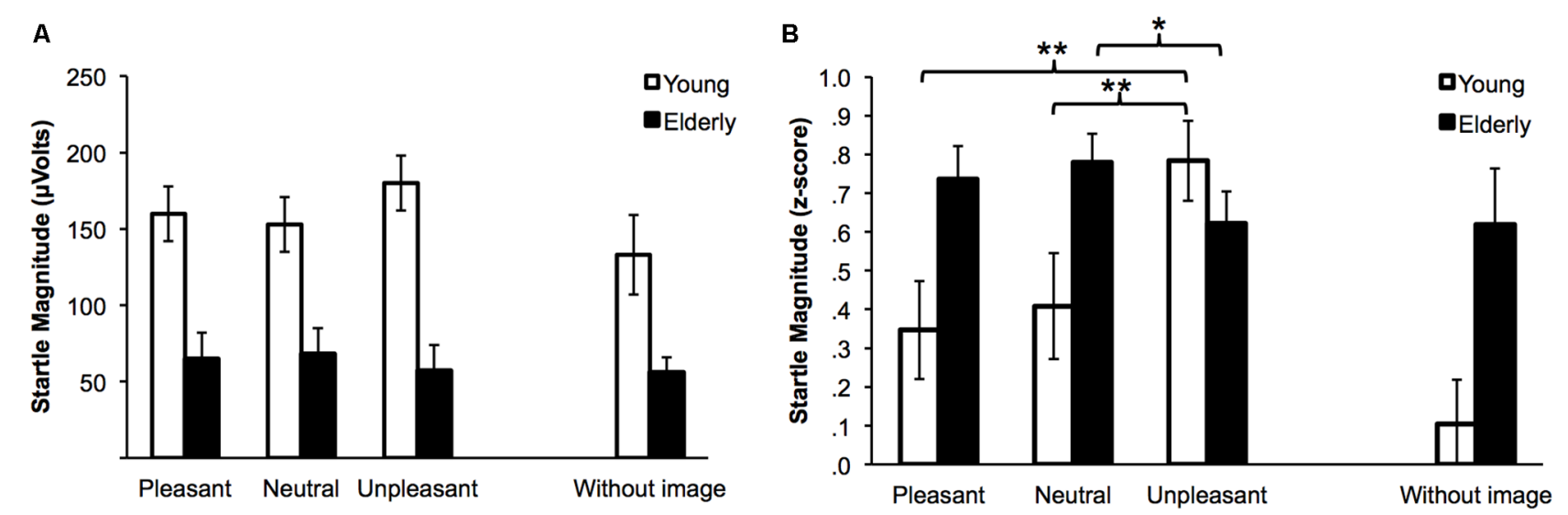

FIGURE 2 | Mean raw magnitude (A) and normalized data (B) of startle blink responses while viewing pleasant, neutral and unpleasant images for Young adults and Elderly. The without-image condition is shown for comparison. Error bars are standard errors. ${ }^{*} p<0.05 ;{ }^{* *} p<0.01$. 
TABLE 2 | Latency of the startle reflex and latency facilitation of the prepulse inhibition values (in ms) for all emotional conditions for both groups.

\begin{tabular}{|c|c|c|c|c|c|}
\hline \multirow[t]{2}{*}{ Measures } & \multirow[t]{2}{*}{ Groups } & \multicolumn{4}{|c|}{ Conditions } \\
\hline & & Pleasant & Neutral & Unpleasant & No image \\
\hline \multirow[t]{2}{*}{ Startle Latency in ms (SD) } & Elderly & $* 67$ (7) & $64(8)$ & 64 (8) & $65(9)$ \\
\hline & Young & *59 (5) & $57(7)$ & 57 (4) & $61(7)$ \\
\hline \multirow[t]{2}{*}{ Latency facilitation in ms (SD) } & Elderly & $11(9)$ & $5(9)$ & $7(14)$ & $6(12)$ \\
\hline & Young & $3(8)$ & $3(8)$ & $3(7)$ & $6(7)$ \\
\hline
\end{tabular}

*Startle latency is significantly longer for pleasant images than neural and unpleasant.

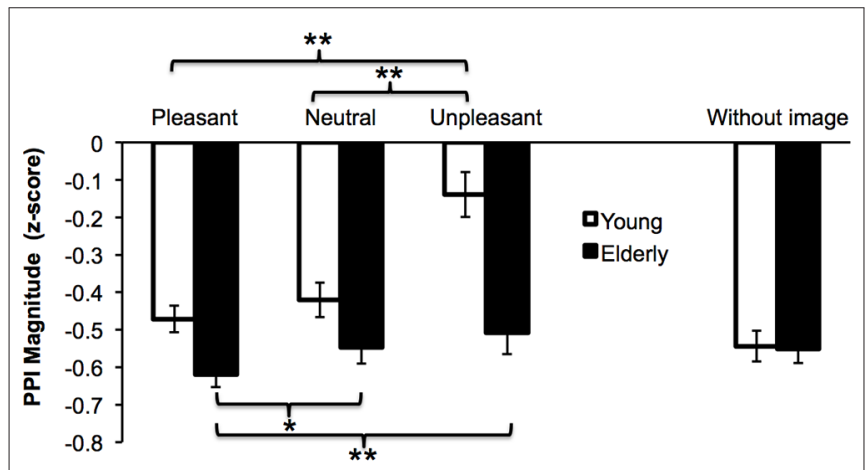

FIGURE 3 | Mean normalized PPI magnitude prepulses while viewing pleasant, neutral and unpleasant images for Younger adults and Elderly. The without-image condition is shown for comparison. Error bars are standard errors. ${ }^{*} p<0.05 ;{ }^{* *} p<0.01$.

\section{Startle Latency}

The Elderly group displayed overall longer latencies compared to Young adults, as supported by a main Group effect, $F_{(1,53)}=30.8$, $p<0.001, \eta_{p}^{2}=0.368$ (Young: $M=58 \mathrm{~ms}, \mathrm{SD}=4$, Elderly: $M=65 \mathrm{~ms}, \mathrm{SD}=6)$. A main effect of Emotion, $F_{(2,106)}=4.24$, $p=0.017, \eta_{p}^{2}=0.074$, revealed that irrespective of the group latency was greater for pleasant pictures $(M=63 \mathrm{~ms}, \mathrm{SD}=7 \mathrm{~ms})$ than neutral $\left(M=61 \mathrm{~ms}, \mathrm{SD}=8 \mathrm{~ms}, t_{(54)}=2.68, p=0.015\right)$ and unpleasant pictures $\left(M=61 \mathrm{~ms}, \mathrm{SD}=7 \mathrm{~ms}, t_{(54)}=2.51\right.$, $p=0.01)$. For the no image condition, there was a trend towards a slower reflex in the elderly but the difference was not significant $t_{(53)}=-1.72, p=0.092$.

\section{Latency Facilitation}

On latency facilitation the three-way ANOVA revealed a significant main effect of Group, $F_{(1,52)}=6.67, p=0.013$, $\eta_{p}^{2}=0.114$, with the Elderly group displaying greater latency facilitation (that is, greater shortening of latency because of the prepulse) than Young adults (Elderly: $M=8 \mathrm{~ms}, \mathrm{SD}=6 \mathrm{~ms}$; Young: $M=3 \mathrm{~ms}, \mathrm{SD}=5 \mathrm{~ms}$ ). A significant two-way interaction between Frequency and Group also emerged, $F_{(1,52)}=4.40$, $p=0.041, \eta_{p}^{2}=0.078$. For low frequency only, the Elderly showed greater latency facilitation $(M=9 \mathrm{~ms}, \mathrm{SD}=9 \mathrm{~ms})$ than Young adults $(M=2 \mathrm{~ms}, \mathrm{SD}=7 \mathrm{~ms}), t_{(52)}=-3.14$, $p=0.003$.

In order to examine the effect of sex as a confound variable, the analyses were repeated using sex as a covariate. There were no main effect of Sex or interaction with Sex for either startle reactivity or PPI (all Fs $<1$ ). The same results (i.e., no effect) was obtained when using Hearing thresholds (low, mid, or high PTAs; all Fs $\leq 1$ ).

\section{DISCUSSION}

Main findings are as follows: (1) emotions modulate sensorimotor gating abilities as assessed by the PPI paradigm; (2) the influence of emotions is different among younger compared to older adults; and (3) remarkably, such preattentional neurophysiological mechanisms are subject to a positivity bias among the elderly population. Indeed, the elderly displayed increased PPI when a positive state was induced whereas young adults displayed a reduced inhibition under negative state conditions. In both groups, the patterns of PPI responses were very different from the effect of emotion on startle reactivity. In agreement with a previous report (Feng et al., 2011), we found enhanced startle reactivity in young adults and reduced startle reactivity in the elderly when negative states were induced. These findings are not fully accounted for by the motivational priming hypothesis of Lang et al. (1990), that is, that the startle reflex is an aversive or defensive response. Rather, the response seems to be modulated by factors such as age.

The different patterns of response between the PPI and startle reactivity paradigms in both groups argue in favor of two different underlying neurophysiological processes as previously suggested. Hawk and Cook (2000) using a similar paradigm in undergraduate students found increased startle reactivity while participants were viewing negative pictures but without effect of emotion on PPI. One possible explanation for the divergence between their PPI results (the only comparable data available) and ours might lie in the stringent criteria used in the present study since we enforced explicit exclusion criteria known to influence the startle reflex such as smoking, hearing thresholds and depression, whereas Hawk and Cook (2000) did not specify any exclusion criteria based on health or lifestyle. More importantly, only participants having a 100\% response probability for startle trials were included in our analyses, i.e., only those with a startle reflex on every non-rejected startle trials ( $<2$ SD above baseline). This objective criterion was used to maximize the probability of finding an effect of emotion on startle reactivity (which we did, similarly to Feng et al., 2011) and PPI, both paradigms being investigated - for the first time-in all participants in the present study. Since groups differed in startle reactivity (older adults had smaller blinks 
than younger ones), this criterion ensured that our study was adequately powered with similar numbers of trials to detect differences between groups. Having examined both types of responses in the same individuals, we are therefore confident in the robustness of our findings. At the same time, the necessity of using such a strict criterion highlights methodological limitations of the startle and PPI techniques to assess emotional influence on sensorimotor gating process. Auditory evoked potentials could be used in follow-up studies looking at the interaction of emotion and aging on sensory gating process to avoid these limitations (Broyd et al., 2013; Smith et al., 2013).

Both sensorimotor gating and emotional processing seem to be well preserved in aging, as shown by similar levels of PPI in the no-image condition in young and older adults, and a strong effect of emotion regulation by the prepulse. Older adults have a bias towards positive emotional stimuli and decreased processing of negative emotional stimuli (the positivity effect, Mather and Carstensen, 2005), they remember more positive than negative information than young people (Charles et al., 2003) and display reduced late positive potential (LPP) amplitude for negative stimuli (Mathieu et al., 2014). When free to look at positive, neutral, or negative pictures, a higher proportion of pictures remembered by older adults consisted of positive than negative information compared with the younger adults. Older adults also display more fixations and of longer duration for positive compared to negative or neutral pictures, a pattern different from younger adults (Sasse et al., 2014). A recent meta-analysis including 100 empirical studies on the positivity effect showed that the effect is robust across a wide range of tasks and stimuli (Reed et al., 2014). Here, we add new data showing that this positivity effect can be shown at pre-attentive stages. A few brain imaging studies have also shown that older adults display greater amygdala activity for positive vs. negative pictures (Mather et al., 2004; Mather and Carstensen, 2005). Since both startle reflexes and PPI can be modulated by input from the amygdala (Koch, 1999), it is likely that the amygdala is one important relay for the effect described here, and that this effect is more easily observable through the simpler neural

\section{REFERENCES}

Beck, A. T., Steer, R. A., and Brown, G. K. (1996). Manual for the Beck Depression Inventory-II. San Antonio, TX: Psychological Corporation.

Blumenthal, T. D., Cuthbert, B. N., Filion, D. L., Hackley, S., Lipp, O. V., and van Boxtel, A. (2005). Committee report: guidelines for human startle eyeblink electromyographic studies. Psychophysiology 42, 1-15. doi: 10.1111/j.14698986.2005.00271.x

Bradley, M. M., Cuthbert, B. N., and Lang, P. J. (1990). Startle reflex modification: emotion or attention? Psychophysiology 27, 513-522. doi: 10.1111/j.1469-8986. 1990.tb01966.x

Bradley, M. M., Cuthbert, B. N., and Lang, P. J. (1993). Pictures as prepulse: attention and emotion in startle modification. Psychophysiology 30, 541-545. doi: 10.1111/j.1469-8986.1993.tb02079.x

Bradley, M. M., Cuthbert, B. N., and Lang, P. J. (1996a). Lateralized startle probes in the study of emotion. Psychophysiology 33, 156-161. doi: 10.1111/j.14698986.1996.tb02119.x circuit of the startle reflex. Future studies should look at the contribution of this structure on the processing of emotions on both startle and PPI. A methodological implication of these findings is that since the emotional status can have a significant influence on physiological reactivity, studies looking at the differential effects of age on the acoustic startle and PPI should take into account the emotional state of their participants.

In conclusion, although older adults show a reduced and delayed acoustic startle response compared to young adults (consistent with previous studies, e.g., Kofler et al., 2001; Ellwanger et al., 2003), their responses are strongly modulated by emotions. Older adults display diminished startle reactivity while viewing unpleasant compared to neutral and pleasant images, which is the reverse pattern from younger adults who display a potentiated reflex while viewing unpleasant images compared to neutral and pleasant images. In addition, older adults display stronger sensorimotor gating while viewing positive compared to neutral or unpleasant images, a pattern different from the younger adults. These results argue in favor of a differential emotional influence on the sensorimotor abilities among young and older adults, with a positivity bias among the latter.

\section{AUTHOR CONTRIBUTIONS}

All authors listed, have made substantial, direct and intellectual contribution to the work, and approved it for publication.

\section{ACKNOWLEDGMENTS}

We thank Nathanaël Lécaudé for programming the tasks described in this article. This research was made possible thanks to a Canadian Foundation Innovation (CFI) Grant. JLD was supported by a studentship from NSERC-Create: training in Auditory Cognitive Neuroscience. PF was supported by studentships from Institut de recherche Robert-Sauvé en santé et sécurité du Travail du Québec (IRSST) and from Fonds de recherche Santé du Québec (FRQS). We thank the Centre de recherche de l'Institut Universitaire de Gériatrie de Montréal (CRIUGM) for help in recruiting elderly participants.

Bradley, M. M., Cuthbert, B. N., and Lang, P. J. (1996b). Picture media and emotion: effects of a sustained affective context. Psychophysiology 33, 662-670. doi: 10.1111/j.1469-8986.1996.tb02362.x

Bradley, M. M., Drobes, D., and Lang, P. J. (1996c). A probe for all reasons: reflex and RT measures in perception. Psychophysiology 33, S25-S25. doi: 10. 1037/e526132012-091

Bradley, M. M., and Lang, P. J. (1994). Measuring emotion: the selfassessment mannequin and the semantic differential. J. Behav. Ther. Exp. Psychiatry 25, 49-59. doi: 10.1016/0005-7916(94) 90063-9

Bradley, M. M., and Lang, P. J. (2000). Affective reactions to acoustic stimuli. Psychophysiology 37, 204-215. doi: 10.1111/1469-8986.37 20204

Bradley, M. M., and Lang, P. J. (2007). "The International Affective Picture System (IAPS) in the study of emotion and attention," in Handbook of Emotion Elicitation and Assessment, eds J. A. Coan and J. J. B. Allen (New York, NY: Oxford University Press), 29-46. 
Braff, D., and Geyer, M. (1990). Sensorimotor gating and schizophrenia. Human and animal model studies. Arch. Gen. Psychiatry 47, 181-188. doi: 10. 1001/archpsyc.1990.01810140081011

Braff, D., Geyer, M., and Swerdlow, N. R. (2001). Human studies of prepulse inhibition of startle: normal subjects, patient groups and pharmacological studies. Psychopharmacology 156, 234-258. doi: 10.1007/s002130 100810

Braff, D., Stone, C., Callaway, E., Geyer, M., Glick, I., and Bali, L. (1978). Prestimulus effects on human startle reflex in normals and schizophrenics. Psychophysiology 15, 339-343. doi: 10.1111/j.1469-8986.1978.tb01390.x

Broyd, S. J., Greenwood, L.-M., Croft, R. J., Dalecki, A., Todd, J., Michie, P. T., et al. (2013). Chronic effects of cannabis on sensory gating. Int. J. Psychophysiol. 89, 381-389. doi: 10.1016/j.ijpsycho.2013.04.015

Cadenhead, K. S., Carasso, B. S., Swerdlow, N. R., Geyer, M. A., and Braff, D. L. (1999). Prepulse inhibition and habituation of the startle response are stable neurobiological measures in a normal male population. Biol. Psychiatry 45, 360-364. doi: 10.1016/s0006-3223(98)00294-7

Charles, S. T., Mather, M., and Carstensen, L. L. (2003). Aging and emotional memory: the forgettable nature of negative images for older adults. J. Exp. Psychol. Gen. 132, 310-324. doi: 10.1037/0096-3445.132.2.310

Cook, E. W., III, Hawk, L. W., Jr., Davis, T. L., and Stevenson, V. E. (1991). Affective individual differences and startle reflex modulation. J. Abnorm. Psychol. 100, 5-13. doi: 10.1037/0021-843x.100.1.5

Cuthbert, B. N., Bradley, M. M., and Lang, P. J. (1996). Probing picture perception: activation and emotion. Psychophysiology 33, 103-111. doi: 10.1111/j.14698986.1996.tb02114.x

Ellwanger, J., Geyer, M. A., and Braff, D. L. (2003). The relationship of age to prepulse inhibition and habituation of the acoustic startle response. Biol. Psychol. 62, 175-195. doi: 10.1016/s0301-0511(02)00126-6

Feng, M. C., Courtney, C. G., Mather, M., Dawson, M. E., and Davison, G. C. (2011). Age-related affective modulation of the startle eyeblink response: older adults startle most when viewing positive pictures. Psychol. Aging 26, 752-760. doi: 10.1037/a0023110

Fournier, P., and Hébert, S. (2013). Gap detection deficits in humans with tinnitus as assessed with the acoustic startle paradigm: does tinnitus fill in the gap? Hear. Res. 295, 16-23. doi: 10.1016/j.heares.2012.05.011

Grillon, C., and Baas, J. (2003). A review of the modulation of the startle reflex by affective states and its application in psychiatry. Clin. Neurophysiol. 114, 1557-1579. doi: 10.1016/s1388-2457(03)00202-5

Grühn, D., and Scheibe, S. (2008). Age-related differences in valence and arousal ratings of pictures from the International Affective Picture System (IAPS): do ratings become more extreme with age? Behav. Res. Methods 40, 512-521. doi: $10.3758 / \mathrm{brm} .40 .2 .512$

Hawk, L. W., and Cook, E. W., III (1997). Affective modulation of tactile startle. Psychophysiology 34, 23-31. doi: 10.1111/j.1469-8986.1997.tb02412.x

Hawk, L. W., and Cook, E. W., III (2000). Independence of valence modulation and prepulse inhibition of startle. Psychophysiology 37, 5-12. doi: 10.1111/14698986.3710005

Koch, M. (1999). The neurobiology of startle. Prog. Neurobiol. 59, 107-128. doi: 10. 1016/S0301-0082(98)00098-7

Kofler, M., Muller, J., Reggiani, L., and Valls-Solé, J. (2001). Influence of age on auditory startle responses in humans. Neurosci. Lett. 307, 65-68. doi: 10. 1016/s0304-3940(01)01908-5

Kumari, V., Fannon, D., Sumich, A. L., and Sharma, T. (2007). Startle gating in antipsychotic-naïve first episode schizophrenia patients: one ear is better than two. Psychiatry Res. 151, 21-28. doi: 10.1016/j.psychres.2006.09.013

Lang, P. J. (1995). The emotion probe. Studies of motivation and attention. Am. Psychol. 50, 372-385. doi: 10.1037/0003-066x.50.5.372
Lang, P. J., Bradley, M. M., and Cuthbert, B. N. (1990). Emotion, attention and the startle reflex. Psychol. Rev. 97, 377-395. doi: 10.1037/0033-295x. 97.3.377

Li, L., Du, Y., Li, N., Wu, X., and Wu, Y. (2009). Top-down modulation of prepulse inhibition of the startle reflex in humans and rats. Neurosci. Biobehav. Rev. 33, 1157-1167. doi: 10.1016/j.neubiorev.2009.02.001

Mather, M., Canli, T., English, T., Whitfield, S., Wais, P., Ochsner, K., et al. (2004). Amygdala responses to emotionally valenced stimuli in older and younger adults. Psychol. Sci. 15, 259-263. doi: 10.1111/j.0956-7976.2004.00662.x

Mather, M., and Carstensen, L. L. (2005). Aging and motivated cognition: the positivity effect in attention and memory. Trends Cogn. Sci. 9, 496-502. doi: 10. 1016/j.tics.2005.08.005

Mathieu, N. G., Gentaz, E., Harquel, S., Vercueil, L., Chauvin, A., Bonnet, S., et al. (2014). Brain processing of emotional scenes in aging: effect of arousal and affective context. PLoS One 9:e99523. doi: 10.1371/journal.pone.00 99523

Patrick, C. J., Bradley, M. M., and Lang, P. J. (1993). Emotion in the criminal psychopath: startle reflex modulation. J. Abnorm. Psychol. 102, 82-92. doi: 10. 1037/0021-843x.102.1.82

Reed, A. E., Chan, L., and Mikels, J. A. (2014). Meta-analysis of the age-related positivity effect: age differences in preferences for positive over negative information. Psychol. Aging 29, 1-15. doi: 10.1037/a0035194

Roy, M., Mailhot, J.-P., Gosselin, N., Paquette, S., and Peretz, I. (2009). Modulation of the startle reflex by pleasant and unpleasant music. Int. J. Psychophysiol. 71, 37-42. doi: 10.1016/j.ijpsycho.2008.07.010

Sasse, L. K., Gamer, M., Büchel, C., and Brassen, S. (2014). Selective control of attention supports the positivity effect in aging. PLoS One 9:e104180. doi: 10. 1371/journal.pone.0104180

Smith, D. M., Grant, B., Fisher, D. J., Borracci, G., Labelle, A., and Knott, V. J. (2013). Auditory verbal hallucinations in schizophrenia correlate with P50 gating. Clin. Neurophysiol. 124, 1329-1335. doi: 10.1016/j.clinph.2013. 02.004

Swerdlow, N. R., Geyer, M. A., and Braff, D. L. (2001). Neural circuit regulation of prepulse inhibition of startle in the rat: current knowledge and future challenges. Psychopharmacology 156, 194-215. doi: 10.1007/s0021301 00799

Vanman, E. J., Boehmelt, A. H., Dawson, M. E., and Schell, A. M. (1996). The varying time courses of attentional and affective modulation of the startle eyeblink reflex. Psychophysiology 33, 691-697. doi: 10.1111/j.1469-8986.1996. tb02365.x

Vrana, S. R., Spence, E. L., and Lang, P. J. (1988). The startle probe response: a new measure of emotion? J. Abnorm. Psychol. 97, 487-491. doi: 10.1037/0021-843x. 97.4.487

Wang, H., He, J., Zhang, R., Zhu, S., Wang, J., Kong, L., et al. (2012). Sensorimotor gating and memory deficits in an APP/PS1 double transgenic mouse model of Alzheimer's disease. Behav. Brain Res. 233, 237-243. doi: 10.1016/j.bbr.2012. 05.007

Conflict of Interest Statement: The authors declare that the research was conducted in the absence of any commercial or financial relationships that could be construed as a potential conflict of interest.

Copyright (c) 2016 Le Duc, Fournier and Hébert. This is an open-access article distributed under the terms of the Creative Commons Attribution License (CC BY). The use, distribution and reproduction in other forums is permitted, provided the original author(s) or licensor are credited and that the original publication in this journal is cited, in accordance with accepted academic practice. No use, distribution or reproduction is permitted which does not comply with these terms. 